\title{
US4all (Ultrasound for all)
}

\section{Ioan Sporea}

Department of Gastroenterology and Hepatology, "Victor Babeș” University of Medicine and Pharmacy, Timișoara, Romania

After more than 50 years of the use of ultrasound in medicine, this method has become the most used imaging method in clinical practice.

What are the advantages of this method? The technique is inexpensive (the price of an ultrasound machine starts from 5000 US dollars), it is mobile, it is easy to be used everywhere, patients do not suffer extreme radiation, it can be repeated as many times as required and it can be used by many specialities.

What is new now in this field? Ultrasound has become a "point of care" method. Thus, it is used in the consultation room by many specialists, starting with emergency doctors, continuing with gastroenterologists, rheumatologists, endocrinologists, urologists, pneumologists and so on. Traditionally, only radiologists, usually working in big departments of imaging, used this method. However, early on, cardiologists and gynecologist/obstetricians started to use this simple and efficient technique in their clinical practice.

In the last 10-15 years, ultrasound entered in force in some fields such as in gastroenterology/hepatology, rheumatology or endocrinology. How was this possible? New ultrasound systems are multipurpose, more mobile, with many types of specialized probes, with moderate prices. New generations of young doctors are now trained to use ultrasound in daily practice. These new generations of specialists who use ultrasound will probably replace the sonographers, who perform most ultrasound examina-

Received Accepted

Med Ultrason

2019, Vol. 21, No 3, 215-216

Corresponding author: Prof. Ioan Sporea, MD, PhD

13, Snagov Street, 300482 Timisoara, Romania

Email: isporea@umft.ro

Phone: +40 256309455

Fax: + 40256488003 tions in some geographical areas (United States, United Kingdom, Norway or Denmark).

What are the main advantages of "point of care" ultrasound? It is a clinical ultrasound (the doctor knows the patient's clinical data), it is performed immediately in the examination room (without any waiting time), and the examination's result is integrated in the clinical decision regarding the patient.

Why ultrasound for all (US4all)? Maybe because there are very few specialties in which ultrasound is not useful in daily practice. Nowadays, apart from gynecology/obstetric or cardiology, where ultrasound is a classical part of the exam, it is difficult to imagine some specialties without ultrasound. The tendency in many countries is to introduce ultrasound as a mandatory module for fellows in training in emergency medicine, gastroenterology/hepatology, rheumatology or endocrinology. Training includes theoretical modules and ultrasound training on simulators or human models, and finishes with examining a number of clinical cases.

The next step in this strategy is to offer ultrasound to general practitioners (GP), performed on portable, inexpensive ultrasound systems. Training in ultrasound for GP has a long history in Romania, and, recently, such a course was offered in the Faroe Islands, during which the majority of local GPs were trained to use US in their practice. Furthermore, using the levels of practice proposed long time ago by EFSUMB (European Federation of Societies for Ultrasound in Medicine and Biology) that include a basic level, as well as advanced and expert levels, this method can be offered for many specialties.

How to extend the number of ultrasound users in daily practice? In the last 5-10 years, ultrasound was introduced in medical schools. Training in ultrasound for students became a normal approach in some medical universities. Ultrasound can be used for anatomy and body physiology learning, but mainly for the evaluation of 
pathological conditions. Hand-on sessions are accepted and liked by medical students. Starting from this point, development of US skills during a fellowship is easy.

Important medical universities around the world introduced ultrasound training in their curricula. Medical students are trained in laboratories with many ultrasound systems, by specialized tutors. Such laboratories can be found in USA or Germany (for instance in Heidelberg). Ultrasound for students was also introduced in Romania, mainly in two centers (Cluj-Napoca and Timișoara).

In the last years, not only the number of ultrasound users increased, but also the quality of ultrasound machines improved. Nowadays, almost all US producers develop systems with high resolution, multiple probes, ultrasound with a large variety of sizes, from very small to high tech. The ultraportable ultrasound systems are very light, and have high quality image. Some companies offer only ultrasound probes, that can be connected to standard tablets or smartphones, which can be used for emergency medicine, cardiology, for vascular applications or for abdominal examinations. The price of such small systems has decreased steadily, making them available for everyone, almost everywhere. Such an ultrasound system ("Butterfly"), with a proposed price of 2000 US dollars, can make ultrasound a part of clinical exam everywhere and for everybody!

Adding to the increasing number of ultrasound users, to the improving quality of ultrasound machines, the innovation in this field has transformed ultrasound into a multiparametric tool. Innovations such as contrast enhanced ultrasound (CEUS) or ultrasound based elastography allow a complex evaluation of patients in the same session.

Thus, the future of ultrasound seems to be very bright. The number of users is continuously increasing, the field of applications is enlarging, and the use of this method as a "point of care" technique is now well established.

Promotion of ultrasound in all fields of medicine is a duty for people with a long experience and intense activity in this field. Attracting new people to ultrasound is our daily aim. Ultrasound for all (US4all) can be a logo for many users of this wonderful method.

Professor Ioan Sporea, MD, PhD 